\title{
All - Silicon Carbide Power Modules Based High Performance Inverter for Traction Applications
}

\author{
B. Kalyana Chakravarthy \\ Assoc. Professor, CVR College of Engineering/EEE Department, Hyderabad, India \\ Email: bkalyan@gmail.com
}

\begin{abstract}
The Silicon (Si) IGBT was an enormous positive disruption to the area of Power Electronics in the 1980s. It has been the workhorse of the industry ever since. It is currently available in its $7^{\text {th }}$ generation. The next revolutionary technology is power semiconductor devices made with wide bandgap materials like Silicon Carbide ( $\mathrm{SiC}$ ). SiC devices have higher critical breakdown strength, wider bandgap, thinner drift layer and better heat conductivity when compared to $\mathrm{Si}$ devices. A traction inverter using $\mathrm{SiC}$ devices will have low value of conductivity loss, lower values of switching and recovery losses, increased range in terms of switching frequency and temperature with better heat dissipation. The converter will be smaller and lighter due to less cooling requirements. A study was done on various options available. Efficiency was compared using an improved model of $\mathrm{SiC}$ MOSFET.
\end{abstract}

Index Terms: SiC, traction inverter, compact, efficient, SBD

\section{INTRODUCTION}

A conventional IGBT power module is shown in Fig.1 below. In a Hybrid $\mathrm{SiC}$ power module, the anti-parallel diode is replaced by a $\mathrm{SiC}$ Schottky Barrier Diode (SBD). High speed switching is possible with Hybrid - SiC module as the SBD does not have accumulation carriers. All SiC power module has a SiC MOSFET with SiC SBD connected in anti-parallel. Higher efficiency and faster switching will be possible with all $\mathrm{SiC}$ power module. [2][6]

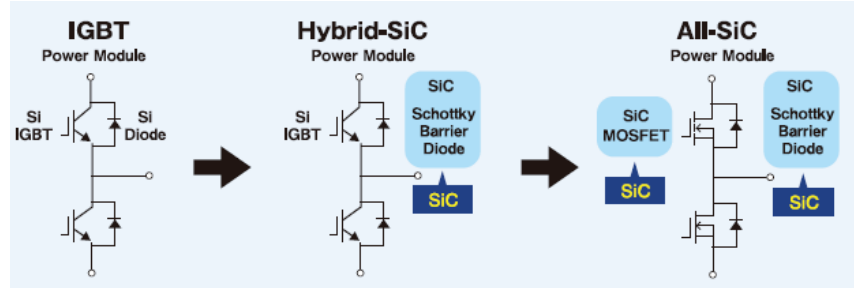

Figure 1. Evolution in power semiconductor device modules

\section{Two-level and three-level traction inverters}

Two-level or three-level inverters are used in most electric traction applications. Two level and three level traction inverters are shown in Fig. 2 and Fig. 3 respectively. These figures show the connections using power modules. Most of the traction inverters are of Variable Voltage Variable Frequency (VVVF) type. Two-level inverter has higher switching loss at higher frequency. The voltage rating of switches used in a three-level inverter is lower than those

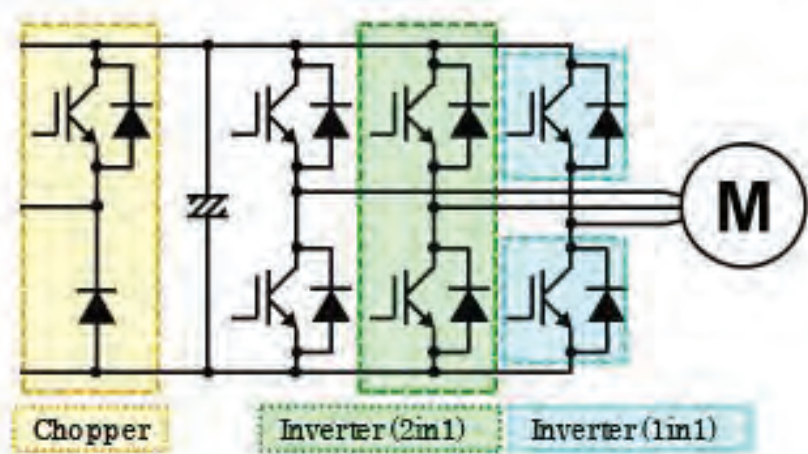

Figure 2. Two - level Traction inverter

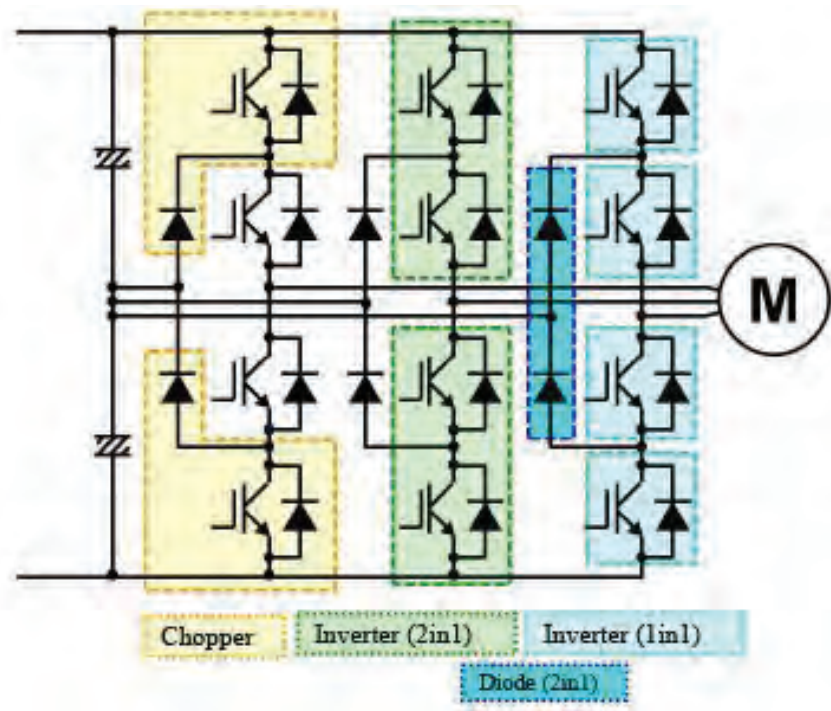

Figure 3. Three - level Traction inverter

used in a two-level inverter for the same DC bus voltage. Switching loss in a 3-level inverter goes down as the switching frequency increases. There is also a reduction in Total harmonic Distortion (THD) due to increase in the number of steps. Reduction in THD leads to less electromagnetic interference (EMI) emission. A 3-level traction inverter [7] connected to a 3-level PWM converter is shown in Fig.4. The control technique used with such traction inverters is usually stator - flux based vector control method. The inverter uses IGBT power modules. A single inverter drives four traction motors in parallel control. A separate traction inverter is used with every traction motor in individual axle control. Reliability of the system is increased when using individual axle control. 


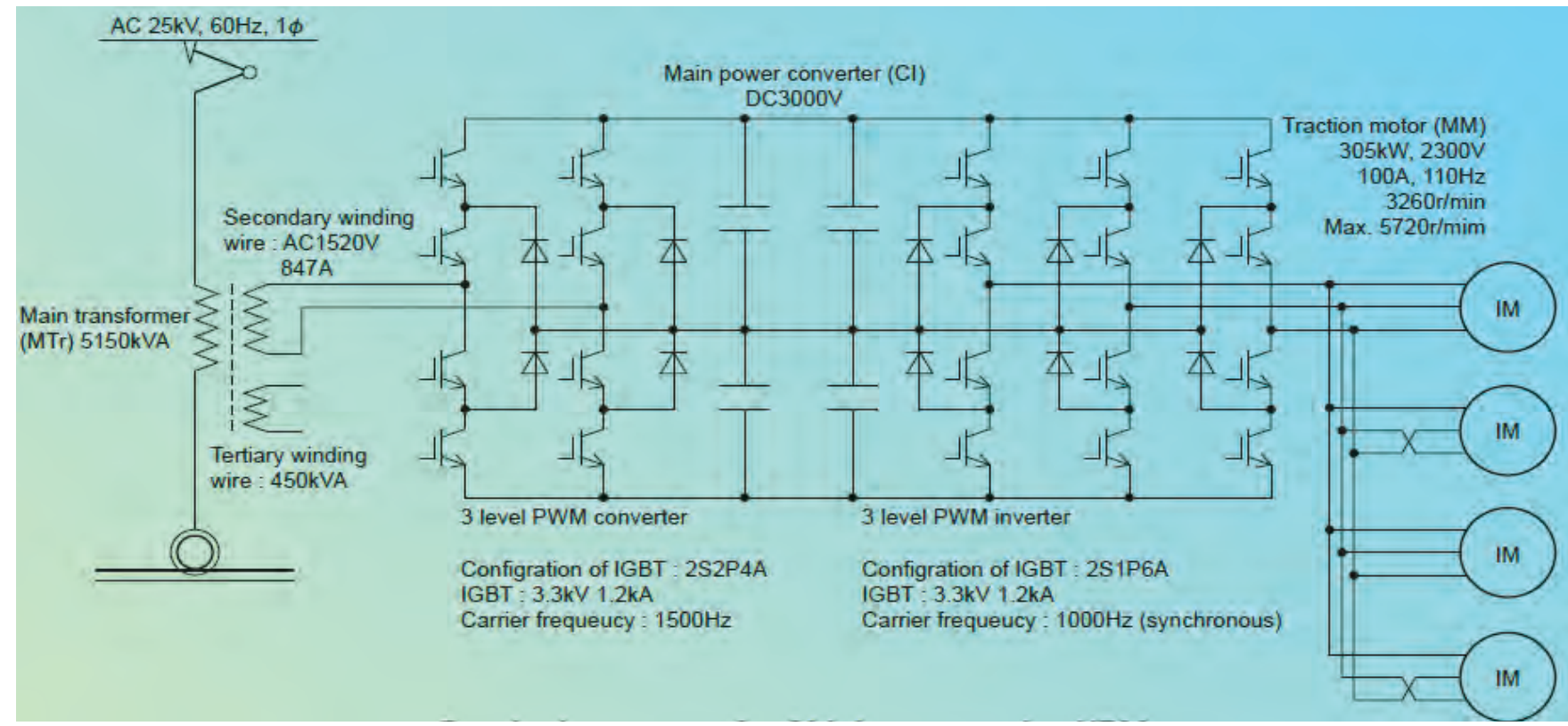

Figure 4. Traction inverter connected to PWM rectifier.

\section{ALL - SiC INVERTER}

\section{A. Replacing power modules}

If the IGBT power modules of converter and inverter in Fig. 4 are replaced by All SiC modules shown in Fig.5, a compact, lightweight propulsion converter with higher efficiency [3] can be realized.
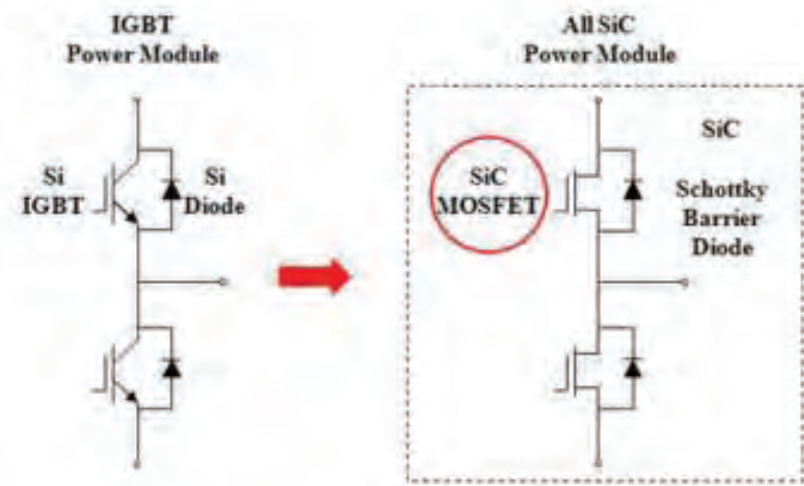

Figure 5. Replacing IGBT power module with All-SiC power module.

All - SiC power module can be switched at a higher switching frequency, which result in higher efficiency, smaller heat-sinks and increased power density. Smaller passive filters are needed when the inverter is operated at higher switching frequency. The All-SiC module is more robust than a conventional IGBT module as it can be operated at higher temperatures. Depending on the operating condition, a 3-level converter may cause a voltage imbalance at the positive and negative sides of the filter capacitor with respect to the DC neutral point. There is a risk that an excessive voltage might be applied to certain components. A technique for neutral point potential control is needed here. When vector control is employed in a single inverter driving many motors, the phase angle of the primary flux is the common state variable. The block diagram of controller for the inverter and converter of Fig.4 is shown in Fig.6.

\section{B. Modulation techniques and controller}

Most of the traction inverters use a modulation technique that changes with speed as shown in Fig.7. Asynchronous PWM is used in low speed range, while synchronous PWM is used in medium speed range. Synchronous single pulses are applied in the high-speed range. A new and energy efficient [1] PWM control technique is shown in Fig. 8. The speed range of asynchronous PWM control has been expanded. PWM control is now based on line voltage control. The carrier frequency is optimized to inhibit lower order harmonics. Low distortion synchronous PWM control is used in the upper end of medium speed range. Lower order harmonics are inhibited, and current harmonics will be reduced. Synchronous triple pulse traction system in the high -speed range also reduces lower order harmonics.

In induction machines, it is desirable to operate the machine at high flux levels allowing the machine to produce higher torques. Estimation of stator flux does not require the knowledge of magnetic circuit parameters. Hence stator flux-based control is more robust and easier to implement in the saturation region than rotor flux-based control. Superior performance, functions and efficiency can be achieved by combining All-SiC based inverter, a controller that uses Reduced Instruction Set Computer (RISC), Digital Signal Processing (DSP) and optic fiber for input and output interface. A $3.3 \mathrm{KV} / 1500$ A power module with SiC MOSFET connected in parallel with a $3.3 \mathrm{KV} \mathrm{SiC}$ Schottky Barrier Diode (SBD) is currently in use. The MOSFET can block a drain current of more than five times larger than its rated current. 


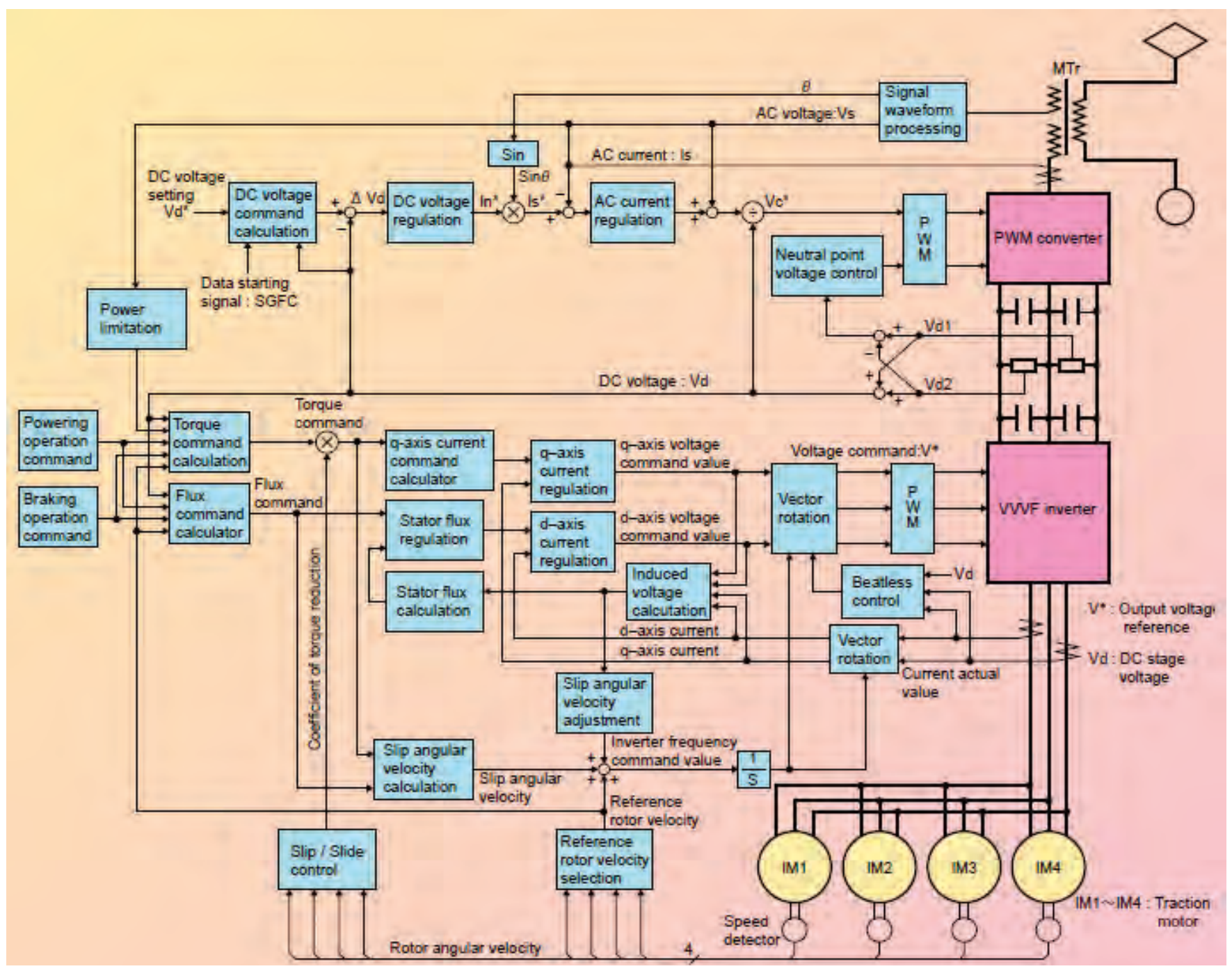

Figure 6. Control block diagram for traction inverter.

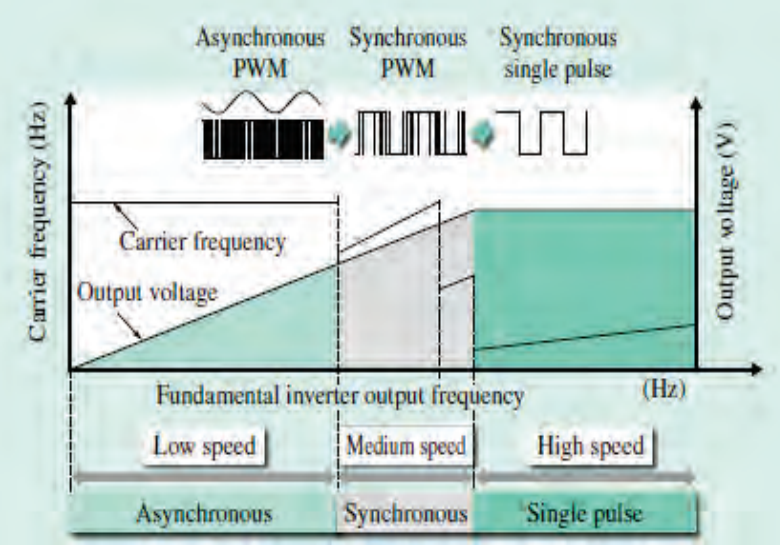

Figure 7. Conventional PWM control technique 


\section{Advantages}

The inverter [2] becomes compact as shown in Fig. 9. This reduction in weight and volume can be attributed to simpler cooling systems, smaller coils and condensers.

\section{Conventional traction inverter with IGBT power modules}

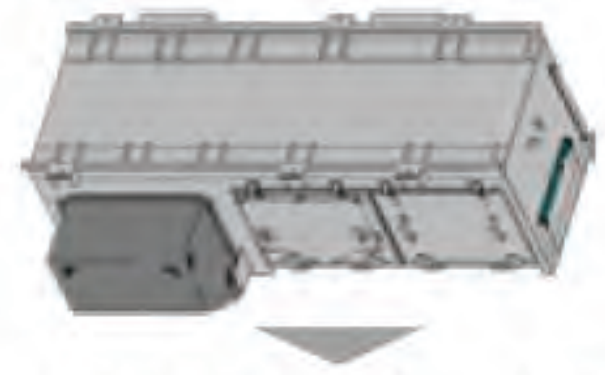

New traction inverter with AllSiC power modules

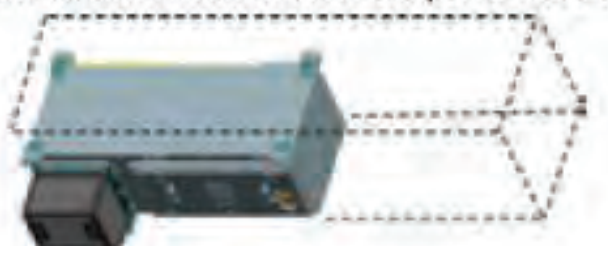

Figure 9. Reduction in the size of inverter

The All-SiC power module has an outstanding advantage as illustrated in Fig.9. The sum of switching and conduction loss remains more or less constant for the entire range of operation. This is not the case for a conventional IGBT power module where the switching loss increases rapidly with increase in switching frequency. This feature helps in recovering more energy during regeneration. As illustrated in Fig. 11 and Fig. 12.

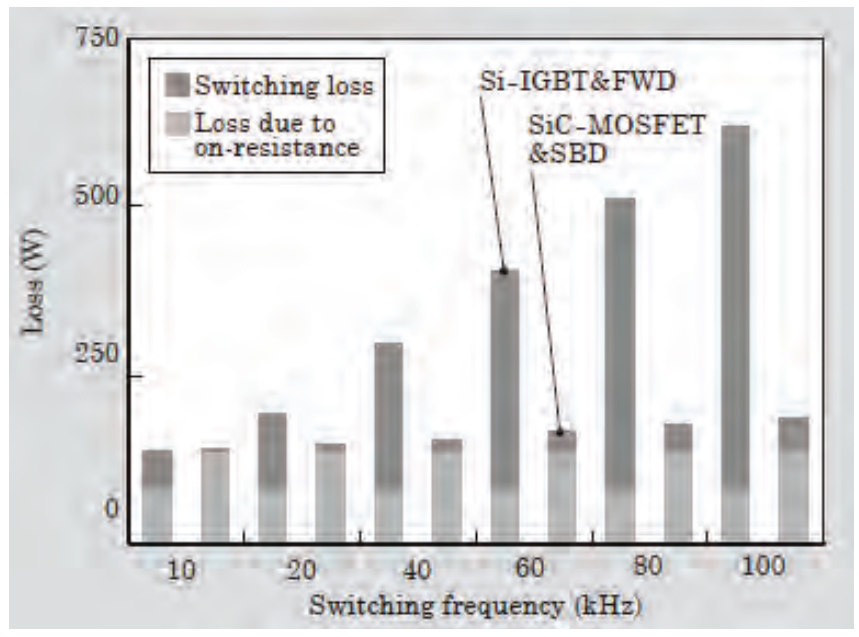

Figure 10. Losses as a function of switching frequency

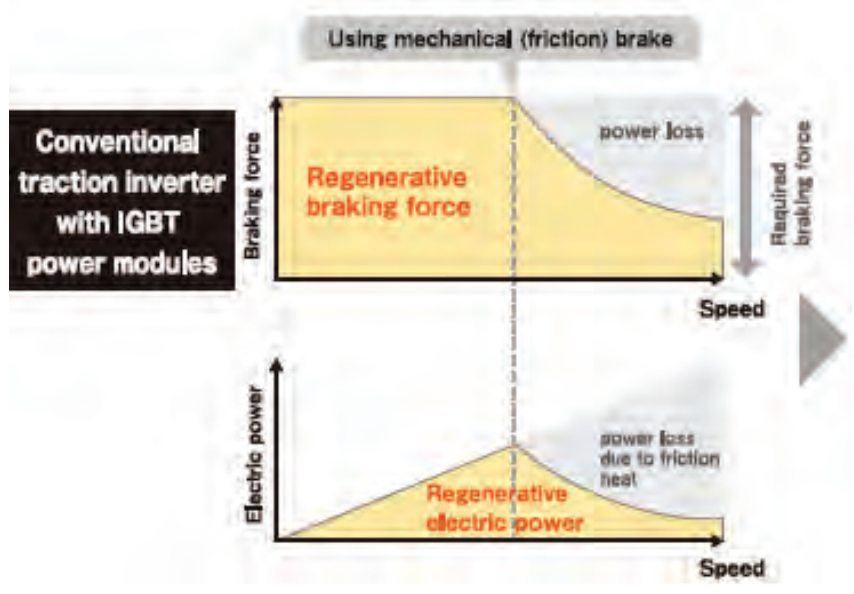

Figure 11. Regenerative braking with inverter using IGBT power modules

\section{Low-power-loss, All-SiC power module applied}
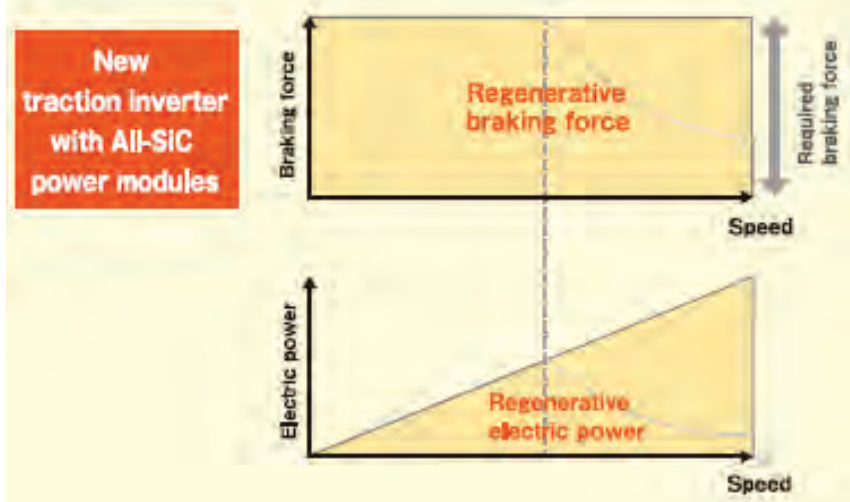

Figure 12. Regenerative braking with inverter using all $\mathrm{SiC}$ power modules

\section{Efficiency comparision}

Efficiency [5] is compared for a three-phase inverter with different power modules. An improved model [4] is considered for All - SiC power MOSFET.

Topology: Three phase inverter

Synchronous rectification ( $\mathrm{SiC}$ version)

DC-link voltage: 400V DC

Current 480Arms (peak) 230Arms (nom)

Switching frequency: $16 \mathrm{kHz}$

$\mathrm{Vgs}=+20 \mathrm{~V} /-5 \mathrm{~V}$ for $\mathrm{SiC}, \mathrm{Vge}= \pm 15 \mathrm{~V}$ for IGBT

PF: 0.8

Modulation index (MI): 1 
The losses, efficiency and current vary as shown in Fig.13 Fig. 14 and Fig. 15 respectively. The All - SiC based inverter had 75 percent lower loss [5] than a conventional IGBT based inverter. Practically [6] the All - SiC based inverter had 40 percent lower loss. Fig. 16 wider operating area of All - SiC power module comparing it with a conventional $\mathrm{Si}$ base power module. The All - SiC module can operate at higher switching frequency and temperature with lower losses.

\section{Inverter losses vs \%load}

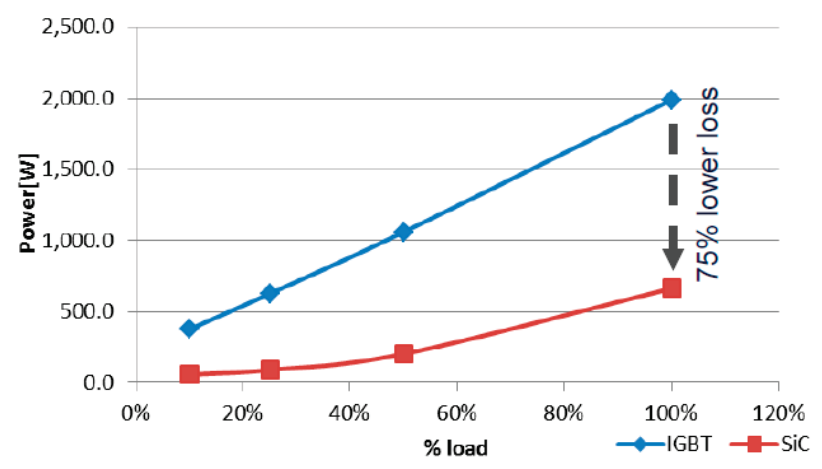

Figure 13. Loss in inverter as a function of load

\section{Inverter efficiency vs \%load}

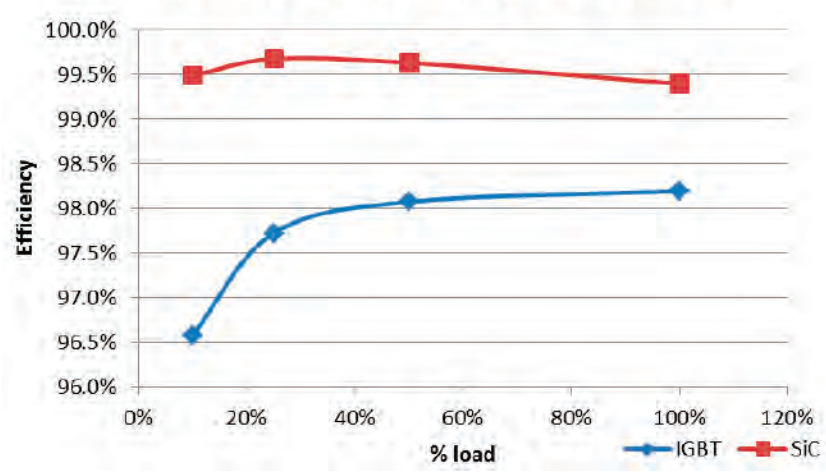

Figure 14. Efficiency of inverter as a function of load.

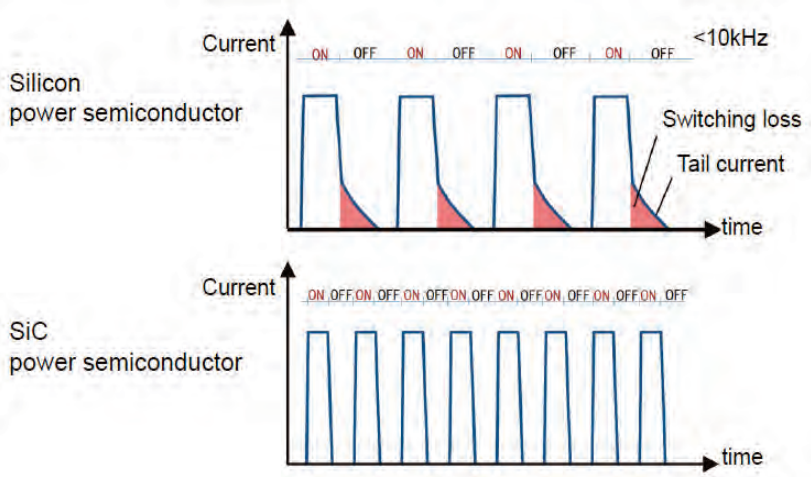

Figure 15. Current waveforms

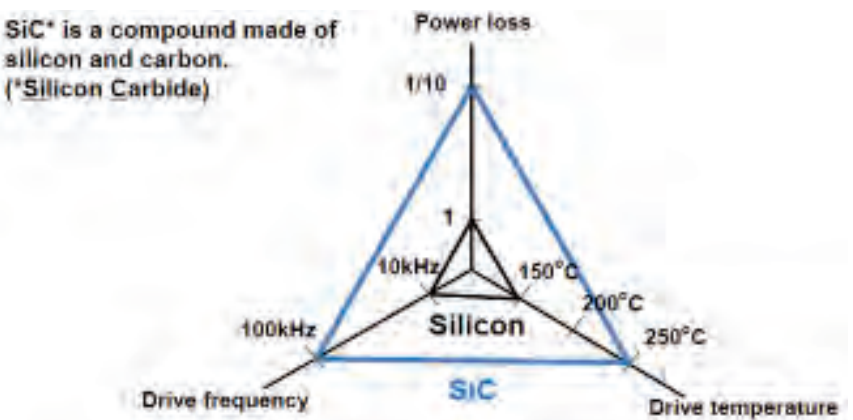

Figure 16. Wider operating area of $\mathrm{SiC}$

Mitsubishi has also started to test a silicon carbide traction converter/inverter system on Shinkansen "bullet" trains operated by the Central Japan Railway Company. These are claimed to be the first $3.3 \mathrm{kV}, 1500 \mathrm{~A}$ traction systems to be installed on a high-speed train. Mitsubishi has been working to shrink traction systems for bullet trains.

The use of the SiC power modules reduces the size of the converter/inverter system by about 55 percent compared to existing systems - and the weight by about 35 percent. The weight of the traction motor, including the drive system, is reduced by around 15 percent. The inverters are being used to power four $305 \mathrm{~kW}$ traction motors. Mitsubishi developed the power modules - which incorporate three-level PWM inverters with regenerative braking.

\section{Summary of Prototype:}

Main Circuit Converter: Three-level modulated singlephase PWM converter

Inverter: Three-level modulated three-phase VVVF inverter

Device used for Converter: 3,300V -1,200A SiC power semiconductor module

Inverter: $3,300 \mathrm{~V}$ - 1,200A $\mathrm{SiC}$ power semiconductor module

Control system: Four 305-kW motors, parallel control

Cooling system: Natural ventilation cooling system

Cree introduced the industry's first commercially available all-silicon carbide ( $\mathrm{SiC}$ ) six-pack power module in an industry standard $45 \mathrm{~mm}$ package. When replacing a silicon module with equivalent ratings, Cree's six-pack module reduces power losses by 75 percent, which leads to an immediate 70 percent reduction in the size of the heat sink, or a 50 percent increase in power density. The six-pack SiC module unlocks the traditional design constraints associated with power density, efficiency and cost, thereby enabling the designer to create high performance, reliable and low-cost power conversion systems. When compared to state-of-theart silicon modules, the $\mathrm{SiC} 1.2 \mathrm{kV}, 50 \mathrm{~A}$ modules deliver performance equivalent to silicon IGBT modules rated up to 150 A depending upon efficiency requirement and switching frequency. Gate driver ICs suitable for SiC MOSFETs are available from IXYS, Texas Instruments and most recently from Avago. 


\section{CONCLuSions}

Currently lowest possible losses in a traction inverter can be achieved only by using all - SiC power modules. These modules have excellent characteristics in terms of efficiency, switching frequency and operation at high temperature. These modules will gradually replace conventional modules in inverters used in traction.

\section{REFERENCES}

[1] Ishikawa K, Terasawa K, Sakai T, Sugimoto S, Nishino T, "Development of rolling stock inverters using SiC," Hitachi review, vol. 66, pp. 155-159, 2017.

[2] Hamada K, Hino S, Miura N, Watanabe H, Nakata S, "3.3 $\mathrm{KV} / 1500 \mathrm{~A}$ power modules for the world's first all $\mathrm{SiC}$ traction inverter", intl. conf. on solid state mat. and dev, vol. 54, pp.1024-1025, 2014.

[3] Wen X, Fan T, Ning P, Guo Q, "Technical approaches towards ultra-high-power density $\mathrm{SiC}$ inverter in electric vehicle applications," CES trans. elec. veh. appl., vol. 1, pp. 231-233, 2017.

[4] Gang Li, Qiang Gao, Miaoxin Jin, Jiabao Kou, Xiaolu Li, Diaoguo Xu," An improved MATLAB/Simulink model of SiC power MOSFETs," int. pow. elec. and. mot. ctrl. conf, vol. 8, pp.1628-1633, 2016.

[5] www.st.com

[6] www.mitsubishielectric.com

[7] www.fujielectric.co.jp 